(C) 2017 IEEE. Personal use of this material is permitted. Permission from IEEE must be obtained for all other uses, in any current or future media, including reprinting/republishing this material for advertising or promotional purposes, creating new collective works, for resale or redistribution to servers or lists, or reuse of any copyrighted component of this work in other works.

\title{
Providing broadband access to extremely sparse areas
}

\author{
C. Mas Machuca, Senior Member IEEE, E. Grigoreva \\ Technische Universität München, Arcisstr. 21, Munich, Germany \\ Tel: +49(0)89 289 23508, Fax: +49(0)89289 23523, e-mail: \{cmas, e.grigoreva\}@tum.de
}

\begin{abstract}
The telecom arena has become very competitive in dense urban areas where the number of potential customers as well as the requirement of high bandwidth are very important. Sparse areas, on the contrary, are left outside the broadband roll out due to the scarce expected revenues and high predicted investments. This work aims at comparing different architectures combining different technologies from different aspects such as investments, power consumption and delivered bandwidth. A particular case study of a sparse area in Finland is analyzed in detail.
\end{abstract}

Keywords: broadband access, optical access network

\section{INTRODUCTION}

Several national and international communication plans target a significant increase of bandwidth connectivity. One example is the European Commission's strategy plan to achieve 30Mbps or more for all users in 2020 [1]. The goal is to increase the availability and take-up of high capacity networks to achieve the so-called Digital Single Market. For that, three objectives have been defined for 2025: Gigabit connectivity, 5G coverage for all urban areas and offering at least $100 \mathrm{Mbps}$ to all European households. This last objective seems realistic to be implemented in dense urban areas where optical distribution networks (ODNs) are available and hence operators expect low required investments and high revenues. Different alternatives have been proposed in literature to upgrade and migrate existing access networks to offer at least $100 \mathrm{Mbps}$ per user: for both passive [2] and active [3,4] access solutions. However, the problem is to find the best access technology able to offer 100 Mbps per household in rural areas: the sparser the area, the higher the cost per user and hence, the lower (if not negative) expected benefits for the operator.

In this paper we study and compare different alternatives that can be used in sparse areas. The comparison is performed in terms of delivered bandwidth, connection availability, cost per household and energy consumption. A very sparse area in Finland has been used as case study.

The paper is organized as follows: Section II presents different architectures that can be used in sparse areas. Section III presents the comparison for a particular case study and finally Section IV concludes the paper.

\section{BROADBAND ACCESS ARCHITECTURES}

This section gives an overview of the different architectures that can deliver at least 100Mbps to end users. For each architecture, a description of the equipment and infrastructure required is provided. Furthermore, a brownfield scenario based on existing copper network is considered as realistic case for Europe.

\subsection{Fiber To The Home/Building (FTTH/FTTB)}

FTTH is interconnecting every Home or Building to the central office (CO) with optical fiber. Different technologies have been proposed based on Time Division Multiplexing (TDM) (e.g., APON/BPON [5], EPON [6], GPON [7] and XG-PON [8]), Wavelength Division Multiplexing (WDM) (e.g., WR-WDM PON and WS-WDM PON $[9,10]$ ), or Hybrid WDM/TDM solutions [10,11]. All these solutions consider a passive ODN, that is, no active component is installed between $\mathrm{CO}$ and the Optical Network Unit (ONU) at the user. However, in order to increase the reach, other solutions consider active ODN, e.g., a cabinet with an Ethernet Switch. The so-called Active Optical Networks (AONs) have been also considered as broadband access solutions [3,4].

The planning of FTTH/B networks is very dependent on the equipment at the remote node and the number of splitting points. For example, the 10Gbps per wavelength transmitted in a XG-PON is shared by up to $\mathrm{N}$ users when a 1:N power splitter is used. Hence, each user gets up to 10/N Gbps. Since our target is to deliver at least 100Mbps, the splitting ratio should be 1:64 at most. Operators may not have just one 1:64 splitter as shown in Figure 1(a), but may place splitters with lower ratio at different cascading nodes: e.g., use 1:8 at one first node and 1:8 at a second a closer to the user node as shown in Figure 1(b). The placement of these nodes impacts significantly the required fiber layout, especially in rural/sparse areas. 


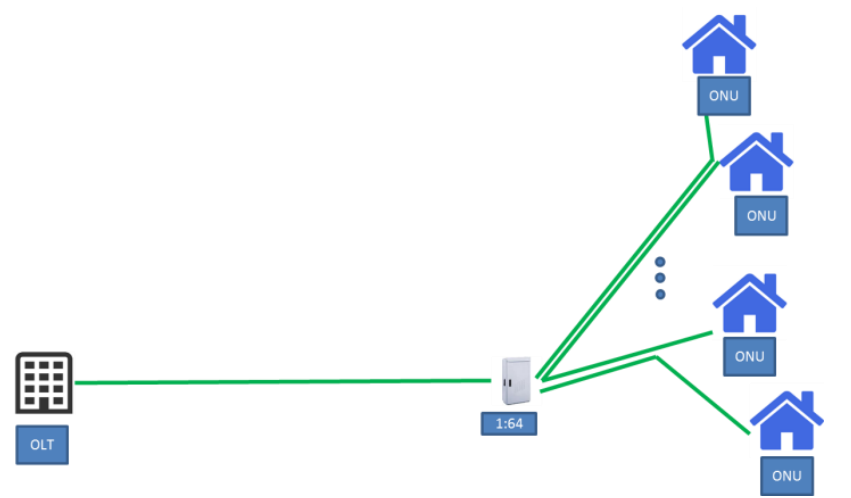

(a) FTTH with one remote node

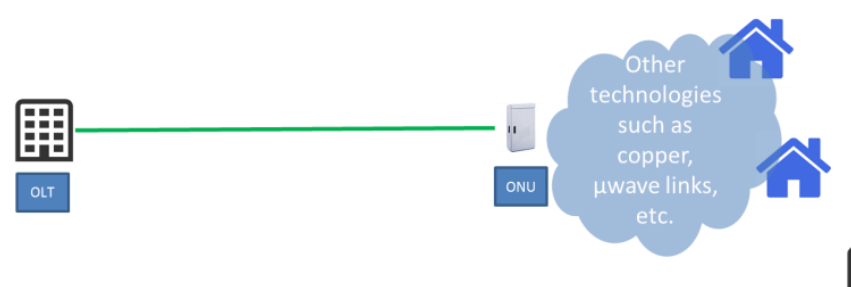

(c) FTTCab P2P

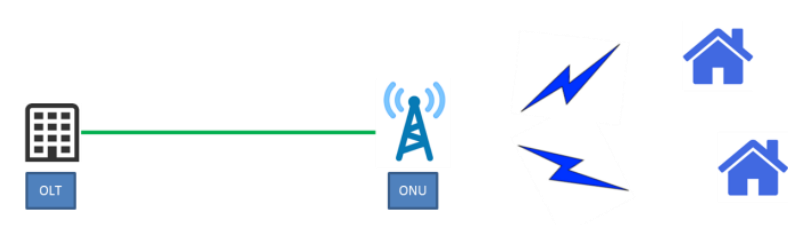

(e) MTT-MBS

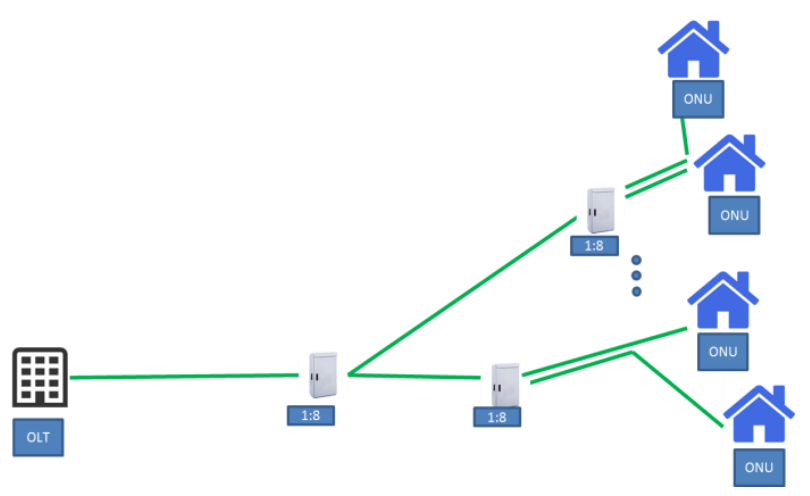

(b) FTTH with two remote nodes

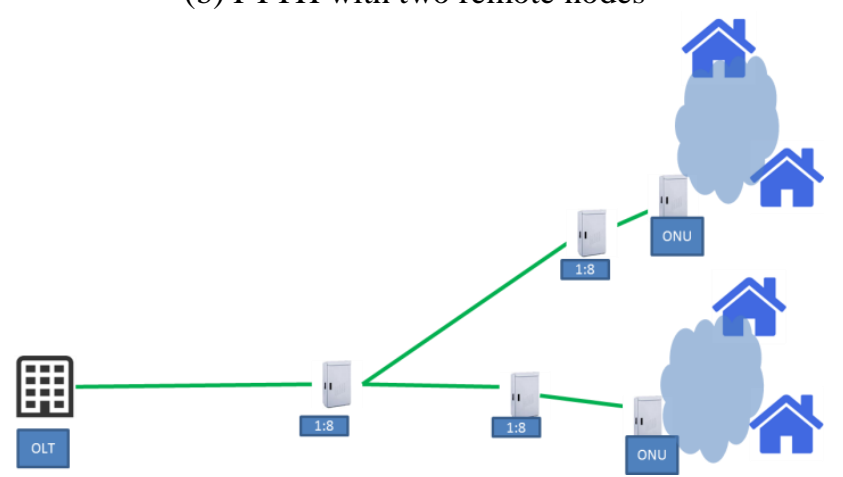

(d) FTTCab with two remote nodes
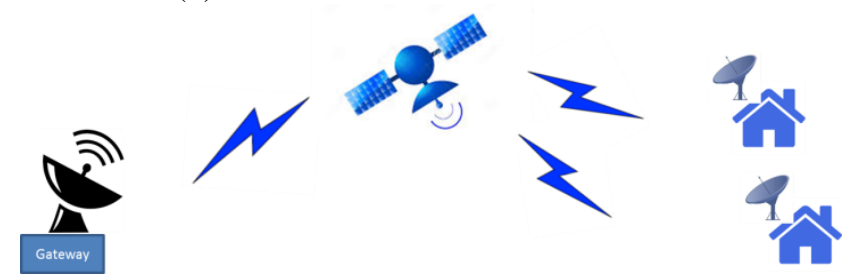

(f) Satellite

Figure 1 Architectures able to deliver at least $100 \mathrm{Mbps}$ per home in sparse areas.

\subsection{Fiber To The Cabinet (FTTCab)}

FTTCab offers a compromise of required investments and delivered bandwidth. Since the targeted delivered bandwidth (100Mbps) can be lower than the delivered by FTTH solutions (up to 500Mbps), it can be shared by several households using other technologies.

1. Copper: copper can transmit $100 \mathrm{Mbps}$ up to 100 meters for CAT5e and $200 \mathrm{~m}$ for CAT6 cables as declared by CustomCable.ca. Using XG-PON allows connecting up to 100 households to the same cabinet, subject to be within $200 \mathrm{~m}$ from the cabinet (it has been shown that using the 17 a vectoring profile, 100Mbps can be offered, and the distance is kept as for ADSL [14]).

2. Radio: wireless communication point to point is possible by using radio equipment, which does not require any additional infrastructure other than the base stations and user equipment. Nowadays, several products allow up to $2 \mathrm{Gbps}$ connections operating at several bands (e.g., E-band [12]). The maximum distance is hence, limited by the band, the area and the meteorological and atmospheric conditions. In this work a maximum distance between building and cabinet of $5 \mathrm{~km}$ as presented in [13] is considered.

\subsection{Satellite}

Satellite networks are suitable for offering connection to large areas since one satellite can around 600 beams, each beam covering an area of $14000 \mathrm{~km}^{2}$ (approx. a circle of $130 \mathrm{~km}$ diameter). In order to calculate the sustainable bandwidth to each user, the capacity from gateway to satellite (equivalent to the Feeder Fiber in FTTH/Cab networks) should be divided among all the beams and all users covered by each beam. So for example, a 1 Tbps from gateway to a satellite of 500 beams, each beam covering 14000 users (assuming very sparse areas with 1 household $/ \mathrm{km}^{2}$ ), each household would get $0,14 \mathrm{Mbps}$, significantly lower than the required bandwidth. In order to increase the delivered bit rate to $100 \mathrm{Mbps}$, one option could be to cover a smaller area and use less beams per 
satellite. If we reduce the covered users from 14000 to 200 and the number of beams from 500 to $50,100 \mathrm{Mbps}$ can be delivered. However, this solution will require more complex antennas at the satellite. Although the delivered bandwidth in satellite systems seems limited, they are very important for resilient purposes in case of disaster or major failure scenario.

\subsection{LTE (Long Term Evolution)}

The LTE roll out considers the placement of the required cells in the area to guarantee coverage and the interconnection either to the central office or aggregation node via $\mu$ wave links or with optical fiber. The number of base station eNodeBs can be calculated from the cell area, which depends on whether the site is omni-directional, bisectoral or tri-sectoral. Let us consider 4G LTE eNodeB from YateBTS which offers 100Mbps downlink capacity sectoral-antenna $50 \mathrm{~m}$ high for less than $10 \mathrm{k} €$. This solution has a coverage from 13,9 to 20,2 $\mathrm{km}^{2}$ (for 3600 to 700 of operating band respectively). This requires a high number of eNodeBs to cover all the rural area.

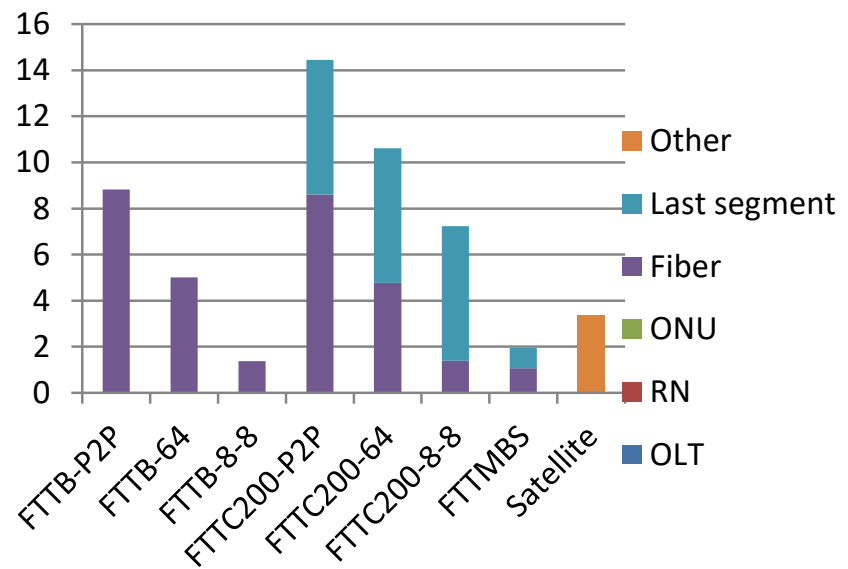

(a) Total cost [Millions €]

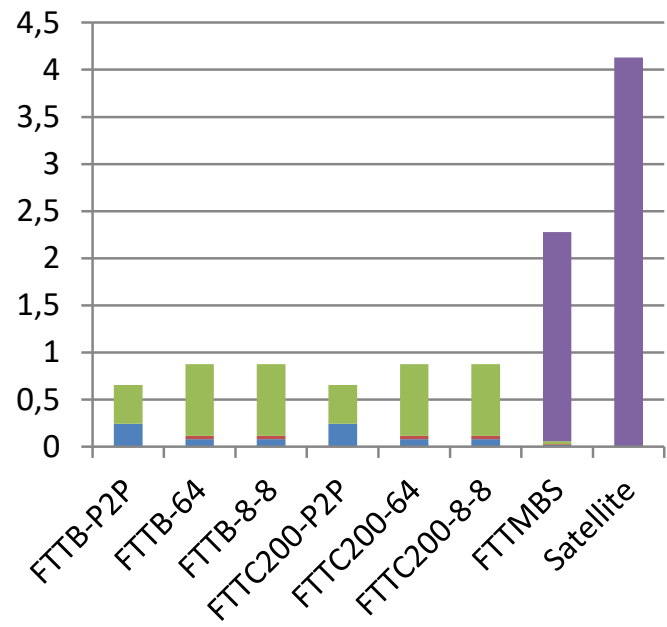

(c) Total power consumption [Thousands Watts]

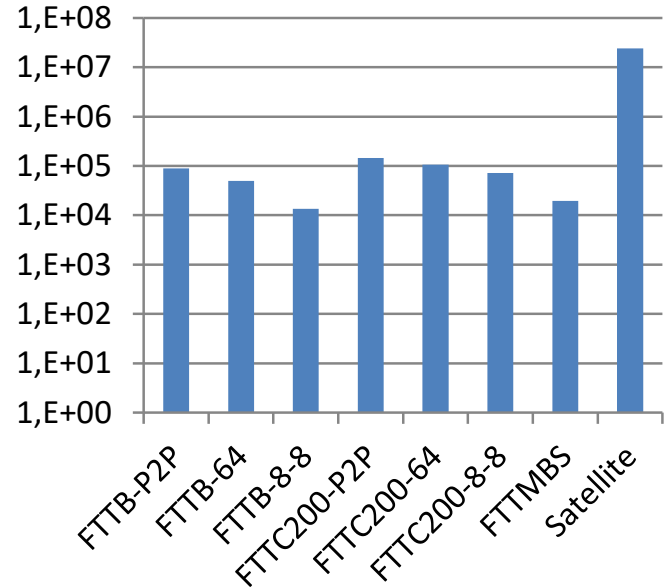

(b) Cost per Mbps [€/Mbps]

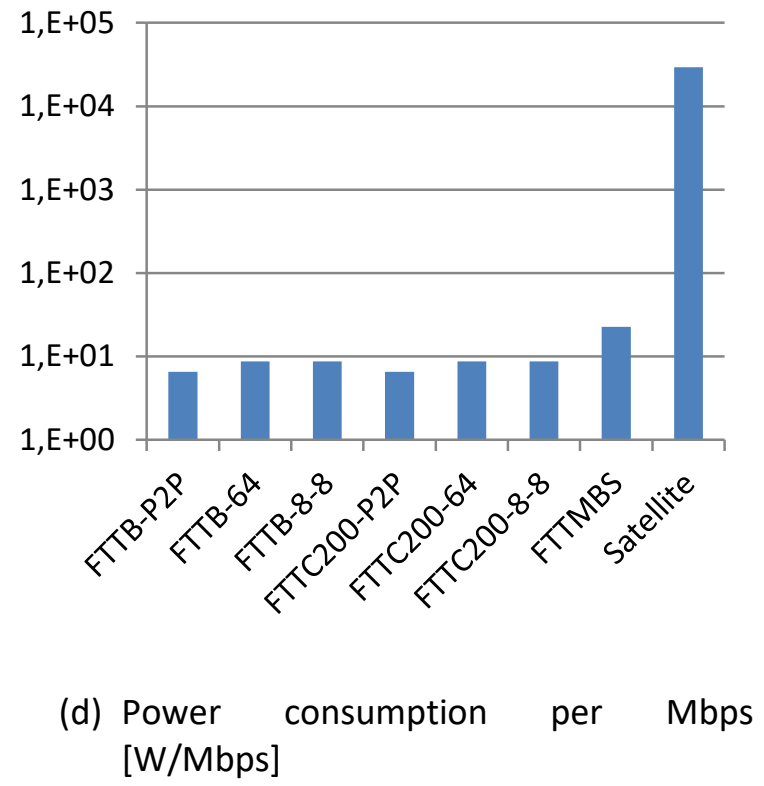

Figure 2 Architecture comparison in terms of cost and power consumtpion

\section{CASE STUDY}

We have considered a very sparsed area in Finland of $1260 \mathrm{~km}^{2}$ with just 117 buildings (i.e., less than a building per $\mathrm{km}^{2}$ ). The goal is to deliver at least $100 \mathrm{Mbps}$ to each building with the different solutions presented in the previous section: 
- "FTTB-P2P": This solution interconnect the Central Office (CO) with each building with dedicated fiber and $100 \mathrm{Mbps}$ transceivers.

- "FTTB-XGPON": This solution uses 10Gbps transceivers to connect up to 64 buildings. Two planning alternatives have been compared: "FTTB-64" considers one single remote node hosting a 1:64 power splitter; and "FTTB-8-8" considers two cascading remote nodes hosting each a 1:8 power spliter. This second alternative promises more flexibility to cluster buildings and less required fiber.

- FTTCx: Existing copper infrastructure can be re-used and the transmitted bandwidth can be increased when using vectoring. It has been shown that up to $200 \mathrm{~m}$, the capacity is not significantly reduced due to copper transmission [14]. Three alternatives have been compared "FTTCx-P2P", "FTTCX-64" and "FTTCx-8-8" following the same nomenclature as for FTTB.

- "FTTMBS": Offering LTE access with the LTE eNodeB mentioned in the previous section, we place the required stations to cover all the buildings within the coverage area. The MBS are then connected point to point to the CO. It has to be mentioned that the delivered bandwidth will be less since the air is a shared

- "Satellite": As alternative to avoid optical fiber installation, we consider the case of satellite. It has been shown that existing solutions offer significantly less bandwidth than the 100Mbps considered in this study.

The comparison of cost and power consumption have been depicted in Figures 2. Although this is just a particular study, some conclusions can be drawn:

- The cost per user are significantly lower for solutions with more remote nodes, which adds the required flexibility in sparse areas.

- Solutions with wirless and satellite solutions have a high penalty in power consumption.

- The savings expected by using FTTC with copper are very low due to the long distances between CO to the cabinets. The savings become even losses when new system such vectoring and copper line has to be installed (considered in this study as last mile).

- Satellite can offer low cost when considering the utitlization of all the beams. However, the delivered bandwidth is low and hence, the cost and power consumption per Mbps is higher than with the other solutions.

- Since the distances are large and the number of intersection points in rural areas are significantly less than in urban and dense urban areas, the savings of FTTC solutions become important when the second technology offers high bit rate with long distances (at least $500 \mathrm{~m}$ ).

- The topology of the area is important in order to gurantee the required Light of Sight need by most of the wireless technolgoies.

\section{REFERENCES}

[1] “A Digital Single Market for Europe: Commission sets out 16 initiatives to make it happen", European Commission - Press release, Brussels, 6 May 2015

[2] C. Mas Machuca, et al., "Methodology for a Cost Evaluation of Migration Toward NGOA Networks" Journal of Optical Communications and Networking, vol. 5, no. 12, pp. 1456-1466, Dec. 2013.

[3] K. Wang et al.," Migration Strategies for FTTx Solutions based on Active Optical Networks”, IEEE Communications Magazine, February 2016.

[4] K. Wang et al., "A Techno-Economic Analysis of Active Optical Network Migration Towards the Next Generation Optical Access", Journal of Optical Communications and Networking, Vol.: 9, Issue: 4, April 2017.

[5] ITU-T Recommendation G.983 ATM and Broadband Passive Optical Networks (APON/BPON)

[6] IEEE 802.3ah standard for Ethernet Passive Optical Networks (EPON)

[7] ITU-T Recommendation G.984 Gigabit-capable Passive Optical Networks (GPON)

[8] ITU-T Recommendation G.987 10Gigabit-capable Passive Optical Networks (XG-PON)

[9] K. Grobe et al., "Combined reach, client-count, power-consumption, and cost analysis of WDM-based next-generation PON" in Eur. Conf. on Optical Communication (ECOC), Switzerland, Sept. 2011

[10] M. Forzati et al., "Next Generation Optical Access Seamless Evolution", Journal of Optical Communications and Networking, pp. 109-123,Vol. 7, 2015.

[11] C. Mas Machuca et al., "Migration from GPON to Hybrid PON: Complete Cost Evaluation", Photonische Netze 2013, Leipzig, May 2013

[12] FibeAir ® IP-20 Platform, Ceragon

[13] J. Wells "The benefits of E-band systems over other wirless technologies", E-band communications Corp., 2010.

[14] Mark Heath "Chart of BT Fibre Broadband FTTC (VDSL2) Speed Versus Distance From the Cabinet", Increase Broadband Speed UK, April 2013 